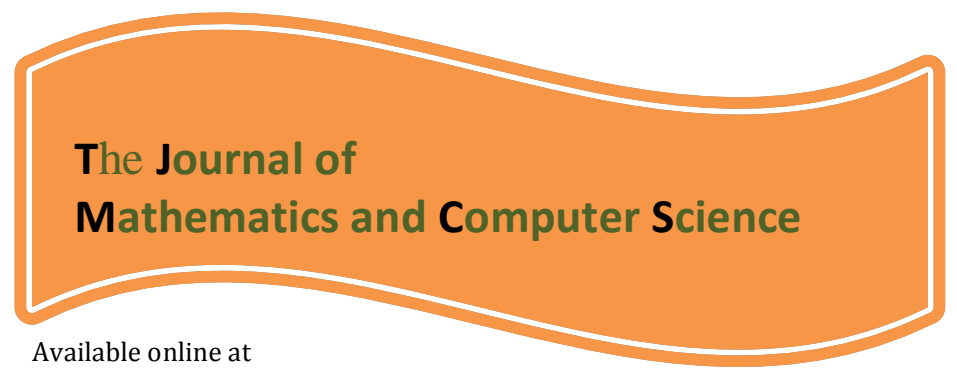

http://www.TJMCS.com

The Journal of Mathematics and Computer Science Vol .3 No.2 (2011) 165 - 175

\title{
Quasi-Permutation Representations for the Borel and Maximal Parabolic Subgroups of $S P\left(4,2^{n}\right)$
}

\author{
M. Ghorbany \\ Department of Mathematics, Iran University \\ of Science and Technology, Emam , Behshahr, Mazandaran , Iran. \\ m-ghorbani@iust.ac.ir
}

Received: May 2011, Revised: June 2011

Online Publication: July 2011

\section{Abstract}

A square matrix over the complex field with non-negative integral trace is called a quasi-permutation matrix.Thus every permutation matrix over $C$ is a quasi-permutation matrix. The minimal degree of a faithful representation of $G$ by quasi-permutation matrices over the complex numbers is denoted by $c(G)$, and $r(G)$ denotes the minimal degree of a faithful rational valued complex character of $G$. In this paper $c(G)$ and $r(G)$ are calculated for the Borel or maximal parabolic subgroups of $S P\left(4,2^{f}\right)$.

Keywords: General linear group, Quasi-permutation.

\section{1- Introduction}

In 1963 Wong defined a quasi-permutation group of degree $n$ to be a finite group $G$ of automorphisms of an $n$-dimensional complex vector space such that every element of $G$ has non-negative integral trace. The terminology drives from the fact that if

2000 AMS Subject Classification:20C15 
$G$ is a finite group of permutations of a set $\Omega$ of size $n$, and we think of $G$ as acting on the complex vector space with basis $\Omega$, then the trace of an element $g \in G$ is equal to the number of points of $\Omega$ fixed by $g$.

Wong studied the extent to which some facts about permutation groups generalize to the quasi-permutation group situation.Then in 1994 Hartley with his colleague investigate further the analogy between permutation groups and quasi-permutation groups by studying the relation between the minimal degree of a faithful permutation representation of a given finite group $G$ and the minimal degree of a faithful quasi-permutation representation. They also worked over the rational field and found some interesting results.(See [2],[8]) .

If $F$ is a subfield of the complex numbers $C$, then a square matrix over $F$ with non-negative integral trace is called a quasi-permutation matrix over $F$.Thus every permutation matrix over $C$ is a quasi-permutation matrix. For a given finite group $G$, let $c(G)$ be the minimal degree of a faithful representation of $G$ by complex quasi-permutation matrices.

By a rational valued character we mean a character $\chi$ corresponding to a complex representation of $G$ such that $\chi(g) \in Q$ for all $g \in G$. As the values of the character of a complex representation are algebraic numbers, a rational valued character is in fact integer valued. A quasi-permutation representation of $G$ is then simply a complex representation of $G$ whose character values are rational and non-negative. The module of such a representation will be called a quasi-permutation module. We will call a homomorphism from $G$ to $G L(n, Q)$ a rational representation of $G$ and its corresponding character will be called a rational character of $G$. Let $r(G)$ denote the minimal degree of a faithful rational valued character of $G$.

If $\varepsilon \in C$ is an algebraic number over $Q$, then the Galois group of $Q(\varepsilon)$ over $Q$ is denoted by $\Gamma$.

Finding the above quantities have been carried out in some papers, for example in [3],[4] , [5] and [7] we found these for the groups $\operatorname{GL}(2, q), \operatorname{SU}\left(3, q^{2}\right), P S U\left(3, q^{2}\right)$, $\operatorname{SL}(3, q), P S L(3, q)$ and $G_{2}\left(2^{n}\right)$ respectively.

In this paper we will calculate $c(G)$ and $r(G)$ for Borel or maximal parabolic subgroups of $\operatorname{SP}\left(4,2^{f}\right)$.

\section{2-Notation and preliminary results}

Assume that $E$ is a splitting field for $G$ and that $F$ is a subfield of $E$. If $\chi, \psi \in \operatorname{Irr}_{E}(G)$ we say that $\chi$ and $\psi$ are Galois conjugate over $F$ if $F(\chi)=F(\psi)$ and there exists $\sigma \in \operatorname{Gal}(F(\chi) / F)$ such that $\chi^{\sigma}=\psi$, where $F(\chi)$ denotes the field obtained by adding the values $\chi(g)$, for all $g \in G$, to $F$. It is clear that this defines an equivalence relation on $\operatorname{Irr}_{E}(G)$.

Let $\eta_{i}$ for $0 \leq i \leq r$ be Galois conjugacy classes of irreducible complex characters

of $G$. For $0 \leq i \leq r$ let $\varphi_{i}$ be a representative of the class $\eta_{i}$, with $\varphi_{o}=1_{G}$. Write 
$\Psi_{i}=\sum_{\chi_{i} \in \eta_{i}} \chi_{i}$ and $K_{i}=k e r \varphi_{i}$. We know that $K_{i}=k e r \Psi_{i}$. For $I \subseteq\{0,1,2, \cdots, r\}$, put $K_{I}=\bigcap_{i \in I} K_{i}$. By definition of $r(G), c(G)$ and using above notations we have:

$r(G)=\min \left\{\xi(1): \xi=\sum_{i=1}^{r} n_{i} \Psi_{i}, n_{i} \geq 0, K_{I}=1\right.$ for $\left.I=\left\{i, i \neq 0, n_{i}>0\right\}\right\}$

$c(G)=\min \left\{\xi(1): \xi=\sum_{i=0}^{r} n_{i} \Psi_{i}, n_{i} \geq 0, K_{I}=1\right.$ for $\left.I=\left\{i, i \neq 0, n_{i}>0\right\}\right\}$

where $n_{0}=-\min \{\xi(g) \mid g \in G\}$.

In [1] we defined $d(\chi), m(\chi)$ and $c(\chi) \quad$ [See Definition 3.4]. Here we can redefine it as follows:

\section{Definition 2.1.}

Let $\chi$ be a complex charater of $G$, such that $\operatorname{ker} \chi=1$ and $\chi=\chi_{1}+\cdots+\chi_{n}$ for some $\chi_{i} \in \operatorname{Irr}(G)$. Then define

(1) $d(\chi)=\sum_{i=1}^{n}\left|\Gamma_{i}\left(\chi_{i}\right)\right| \chi_{i}(1)$,

(2) $m(\chi)= \begin{cases}0 & \text { if } \chi=1_{G}, \\ \left|\min \left\{\sum_{i=1}^{n} \sum_{\alpha \in \Gamma_{i}\left(\chi_{i}\right)} \chi_{i}^{\alpha}(g): g \in G\right\}\right| & \text { otherwise, }\end{cases}$

(3) $c(\chi)=\sum_{i=1}^{n} \sum_{\alpha \in \Gamma_{i}\left(\chi_{i}\right)} \chi_{i}^{\alpha}+m(\chi) 1_{G}$.

So

$$
r(G)=\min \{d(\chi): \operatorname{ker} \chi=1\}
$$

and

$$
c(G)=\min \{c(\chi)(1): \operatorname{ker} \chi=1\} .
$$

We can see all the following statements in [1].

\section{Corollary 2.2.}

Let $\chi \in \operatorname{Irr}(G)$, then $\sum_{\alpha \in \Gamma(\chi)} \chi^{\alpha}$ is a rational valued character of $G$. Moreover $c(\chi)$ is a non-negative rational valued character of $G$ and $c(\chi)=d(\chi)+m(\chi)$.

\section{Lemma 2.3.}

Let $\chi \in \operatorname{Irr}(G), \chi \neq 1_{G}$. Then $c(\chi)(1) \geq d(\chi)+1 \geq \chi(1)+1$.

\section{Lemma 2.4.}

Let $\chi \in \operatorname{Irr}(G)$. Then

(1) $c(\chi)(1) \geq d(\chi) \geq \chi(1)$;

(2) $c(\chi)(1) \leq 2 d(\chi)$. Equality occurs if and only if $Z(\chi) /$ ker $\chi$ is of even order.

\section{Quasi-permutation representations}

We begin with a brief summary of facts relevant to our treatment of the group . 
Let $K$ be the finite field with $q$ elements, where $q=p^{f}$ and $p$ is a prime number. Let $\bar{K}$ be the algebraic closure of $K$, and put

$$
K_{i}=\left\{x \in \bar{K} \mid x^{q^{i}}=x\right\} .
$$

Then $K_{i}$ is the subfield of $\bar{K}$ with $q^{i}$ elements, and $K_{1}=K$. Let $\kappa$ be a fixed generator of the multiplicative group $K_{4}^{*}$ and put $\tau=\kappa^{q^{2}-1}, \theta=\kappa^{q^{2}+1}, \eta=\theta^{q-1}$ and $\gamma=\theta^{q+1}$. Then we have $\langle\theta\rangle=K_{2}^{*}$ and $\langle\gamma\rangle=K^{*}$. Choose a fixed isomorphism from the multiplicative group $K_{4}^{*}$ into the multiplicative group of complex numbers, and let $\widetilde{\tau, \theta, \eta}$ and $\gamma$ be the images of $\tau, \theta, \eta$ and $\gamma$ respectively under this isomorphism. Let $G$ be the 4-dimensional symplectic group over $K$, that is,

$$
G=\left\{\left.A \in G L(4, K)\right|^{t} A J A=J\right\},
$$

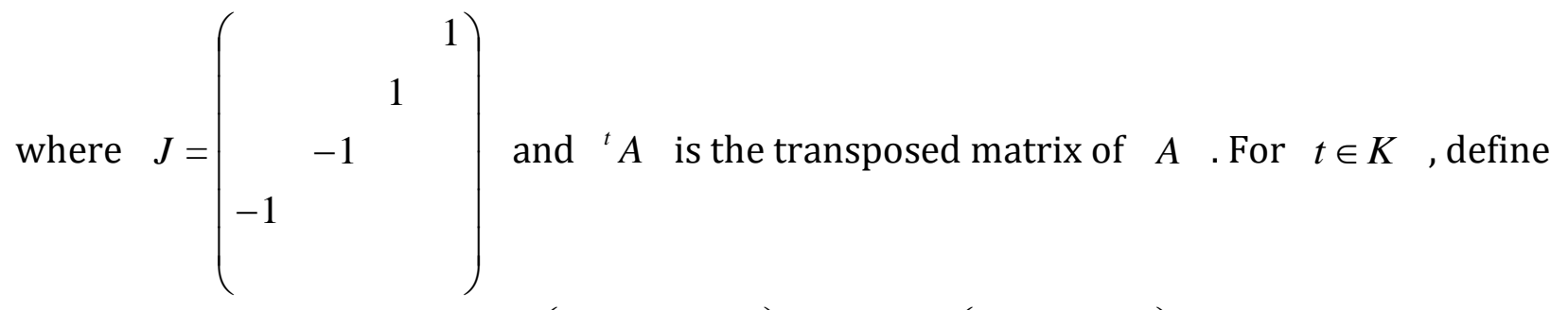
$x_{a}(t)=\left(\begin{array}{cccc}1 & t & & \\ & 1 & & \\ & & 1 & -t \\ & & & 1\end{array}\right), \quad x_{b}(t)=\left(\begin{array}{llll}1 & & & \\ & 1 & t & \\ & & 1 & \\ & & & 1 \\ & & & \end{array}\right)$,

$$
x_{a+b}(t)=\left(\begin{array}{cccc}
1 & & t & \\
& 1 & & t \\
& & 1 & \\
& & & 1
\end{array}\right), x_{2 a+b}(t)=\left(\begin{array}{cccc}
1 & & & t \\
& 1 & & \\
& & 1 & \\
& & & 1 \\
& & &
\end{array}\right) \text {, }
$$

and put $\Delta^{+}=\{a, b, a+b, 2 a+b\}$. Then for $r \in \Delta^{+}, \Xi_{r}=\left\{x_{r}(t) \mid t \in K\right\}$ is a subgroup of $G$ isomorphism to the additive group of $K$, and we have the folloing commutator relations, where the commutator $x^{-1} y^{-1} x y$ is denoted by $[x, y]$ :

$$
\begin{gathered}
{\left[x_{a}(t), x_{b}(u)\right]=x_{a+b}(t u) x_{2 a+b}\left(-t^{2} u\right),} \\
{\left[x_{a}(t), x_{a+b}(u)\right]=x_{2 a+b}(2 t u),} \\
{\left[x_{r}(t), x_{s}(u)\right]=1, \text { for all other pairs of } r, s \in \Delta^{+} .}
\end{gathered}
$$


Next , define $h\left(z_{1}, z_{2}\right)=\left(\begin{array}{cccc}z_{1} & & & \\ & z_{2} & & \\ & & z_{2}^{-1} & \\ & & & z_{1}^{-1}\end{array}\right) \quad$ for $\quad z_{i} \in K_{4}^{*} \quad$ and put $U=\Xi_{a} \Xi_{b} \Xi_{a+b} \times \Xi_{2 a+b}, \wp=\left\{h\left(z_{1}, z_{2}\right) \mid z_{i} \in K^{*}\right\}$ and $B=\wp U$. Then $U$ is a Sylow p-subgroup of $G$, and $B$ is the normalizer of $U$ in $G$ (called the Borel subgroup of $G)$. Put $\omega_{r}=x_{r}(1)^{t} x_{r}(-1) x_{r}(1)$ for $r \in \Delta^{+}$. Especially,

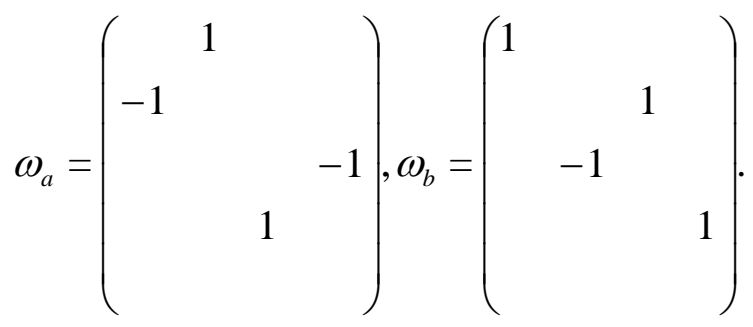

Then $G$ is generated by $B \cup\left\{\omega_{a}, \omega_{b}\right\}$. The maximal parabolic subgroups of $G$ generated by $B \cup\left\{\omega_{a},\right\}$ and $B \cup\left\{\omega_{b},\right\}$ are denoted by $P$ and $Q$ respectively.

We know that every irreducible character of Borel subgroup $B$ is the induced character of some linear character of a subgroup, that is , $B$ is an $M$-group. The character table of $B$ is given in Table $(I)$ and the character tables of $P$ and $Q$ are given in Tables $(I I, I I I)$ of the Appendix of [6].

In the next theorem we shall determine $r(G)$ and $c(G)$ for a Borel subgroup of $\operatorname{SP}\left(4,2^{n}\right)$.

\section{Theorem 3.1.}

Let $G$ be a Borel subgroup of $\operatorname{SP}\left(4,2^{n}\right)$, then

1) $r(B)= \begin{cases}2 m q(q-1) & \text { if } q \geq 4 m+1 \\ \frac{q(q-1)^{2}}{2} & \text { otherwise }\end{cases}$

2) $c(B)=\left\{\begin{array}{ll}2 m q^{2} & \text { if } q \geq 4 m+1, \\ \frac{q^{2}(q-1)}{2} & \text { otherwise }\end{array} \quad\right.$ where $m=\left|\Gamma\left(\chi_{4}(k)\right)\right|$

Proof. By Definition 2.1, in order to calculate $r(G)$ and $c(G)$, we need to determine $d(\chi)$ and $c(\chi)(1)$ for all characters that are faithful or $\bigcap_{\chi} \operatorname{Ker} \chi=1$.

Then by Corollary 2.2, Lemmas 2.3 and 2.4 and Table $(I)$ of [6], for the Borel subgroup $B$ we have:

$d\left(\chi_{1}\right)=\left|\Gamma\left(\chi_{1}(k, l)\right)\right| \chi_{1}(k, l)(1)+\left|\Gamma\left(\theta_{2}(k)\right)\right| \theta_{2}(k)(1) \geq \frac{q(q-1)^{2}}{2}+1$ and $c\left(\chi_{1}\right)(1) \geq 1+\frac{q^{2}(q-1)}{2}$, 


$$
\begin{aligned}
& d\left(\chi_{2}\right)=\left|\Gamma\left(\chi_{2}(k)\right)\right| \chi_{2}(k)(1)+\left|\Gamma\left(\theta_{2}(k)\right)\right| \theta_{2}(k)(1) \geq \frac{(q-1)\left(q^{2}-q+2\right)}{2} \text { and } c\left(\chi_{2}\right)(1) \geq \frac{q\left(q^{2}-q+2\right)}{2} \text {, } \\
& d\left(\chi_{3}\right)=\left|\Gamma\left(\chi_{3}(k)\right)\right| \chi_{3}(k)(1)+\left|\Gamma\left(\theta_{2}(k)\right)\right| \theta_{2}(k)(1) \geq \frac{(q-1)\left(q^{2}-q+2\right)}{2} \text { and } c\left(\chi_{3}\right)(1) \geq \frac{q\left(q^{2}-q+2\right)}{2}, \\
& d\left(\chi_{4}\right)=\left|\Gamma\left(\chi_{4}(k)\right)\right| \chi_{4}(k)(1)+\left|\Gamma\left(\theta_{2}(k)\right)\right| \theta_{2}(k)(1) \geq \frac{q\left(q^{2}-1\right)}{2} \text { and } c\left(\chi_{4}\right)(1) \geq \frac{q^{2}(q+1)}{2} \\
& d\left(\chi_{5}\right)=\left|\Gamma\left(\chi_{5}(k)\right)\right| \chi_{5}(k)(1)+\left|\Gamma\left(\theta_{2}(k)\right)\right| \theta_{2}(k)(1) \geq \frac{q\left(q^{2}-1\right)}{2} \text { and } c\left(\chi_{5}\right)(1) \geq \frac{q^{2}(q+1)}{2}, \\
& d\left(\chi_{6}\right)=\left|\Gamma\left(\theta_{1}\right)\right| \theta_{1}(1)+\left|\Gamma\left(\theta_{2}(k)\right)\right| \theta_{2}(k)(1) \geq \frac{(q-1)^{2}(q+2)}{2} \text { and } c\left(\chi_{6}\right)(1) \geq q^{2}(q-1), \\
& d\left(\chi_{7}\right)=\left|\Gamma\left(\chi_{1}(k, l)\right)\right| \chi_{1}(k, l)(1)+\left|\Gamma\left(\theta_{3}(k)\right)\right| \theta_{3}(k)(1) \geq \frac{q(q-1)^{2}}{2}+1 \text { and } c\left(\chi_{7}\right)(1) \geq 1+\frac{q^{2}(q-1)}{2} \text {, } \\
& d\left(\chi_{8}\right)=\left|\Gamma\left(\chi_{2}(k)\right)\right| \chi_{2}(k)(1)+\left|\Gamma\left(\theta_{3}(k)\right)\right| \theta_{3}(k)(1) \geq \frac{(q-1)\left(q^{2}-q+2\right)}{2} \text { and } c\left(\chi_{8}\right)(1) \geq \frac{q\left(q^{2}-q+2\right)}{2} \text {, } \\
& d\left(\chi_{9}\right)=\left|\Gamma\left(\chi_{3}(k)\right)\right| \chi_{3}(k)(1)+\left|\Gamma\left(\theta_{3}(k)\right)\right| \theta_{3}(k)(1) \geq \frac{(q-1)\left(q^{2}-q+2\right)}{2} \text { and } c\left(\chi_{9}\right)(1) \geq \frac{q\left(q^{2}-q+2\right)}{2} \text {, } \\
& d\left(\chi_{10}\right)=\left|\Gamma\left(\chi_{4}(k)\right)\right| \chi_{4}(k)(1)+\left|\Gamma\left(\theta_{3}(k)\right)\right| \theta_{3}(k)(1) \geq \frac{q\left(q^{2}-1\right)}{2} \text { and } c\left(\chi_{10}\right)(1) \geq \frac{q^{2}(q+1)}{2}, \\
& d\left(\chi_{11}\right)=\left|\Gamma\left(\chi_{5}(k)\right)\right| \chi_{5}(k)(1)+\left|\Gamma\left(\theta_{3}(k)\right)\right| \theta_{3}(k)(1) \geq \frac{q\left(q^{2}-1\right)}{2} \text { and } c\left(\chi_{11}\right)(1) \geq \frac{q^{2}(q+1)}{2}, \\
& d\left(\chi_{12}\right)=\left|\Gamma\left(\theta_{1}\right)\right| \theta_{1}(1)+\left|\Gamma\left(\theta_{3}(k)\right)\right| \theta_{3}(k)(1) \geq \frac{(q-1)^{2}(q+2)}{2} \text { and } c\left(\chi_{12}\right)(1) \geq q^{2}(q-1), \\
& d\left(\chi_{13}\right)=\left|\Gamma\left(\chi_{5}(k)\right)\right| \chi_{5}(k)(1)+\left|\Gamma\left(\chi_{4}(k)\right)\right| \chi_{4}(k)(1) \geq 2 q(q-1) \text { and } c\left(\chi_{13}\right)(1) \geq 2 q^{2}, \\
& d\left(\theta_{2}(k)\right)=\left|\Gamma\left(\theta_{2}(k)\right)\right| \theta_{2}(k)(1)=\frac{q(q-1)^{2}}{2} \text { and } c\left(\theta_{2}(k)\right)(1)=\frac{q^{2}(q-1)}{2} \text {, } \\
& d\left(\theta_{3}(k)\right)=\left|\Gamma\left(\theta_{3}(k)\right)\right| \theta_{3}(k)(1)=\frac{q(q-1)^{2}}{2} \text { and } c\left(\theta_{3}(k)\right)(1)=\frac{q^{2}(q-1)}{2}
\end{aligned}
$$

An overall picture is provided by the Table(I):

Table (I)

\begin{tabular}{|c|c|c|}
\hline$\chi$ & $d(\chi)$ & $c(\chi)(1)$ \\
\hline$\chi_{1}$ & $\geq q(q-1)^{2} / 2+1$ & $\geq 1+q^{2}(q-1) / 2$ \\
\hline$\chi_{2}$ & $\geq(q-1)\left(q^{2}-q+2\right) / 2$ & $\geq q\left(q^{2}-q+2\right) / 2$ \\
\hline$\chi_{3}$ & $\geq(q-1)\left(q^{2}-q+2\right) / 2$ & $\geq q\left(q^{2}-q+2\right) / 2$ \\
\hline$\chi_{4}$ & $\geq q\left(q^{2}-1\right) / 2$ & $\geq q^{2}(q+1) / 2$ \\
\hline$\chi_{5}$ & $\geq q\left(q^{2}-1\right) / 2$ & $\geq q^{2}(q+1) / 2$ \\
\hline$\chi_{6}$ & $\geq(q-1)^{2}(q+2) / 2$ & $\geq q^{2}(q-1)$ \\
\hline
\end{tabular}




\begin{tabular}{|c|c|c|}
\hline$\chi_{7}$ & $\geq q(q-1)^{2} / 2+1$ & $\geq 1+q^{2}(q-1) / 2$ \\
\hline$\chi_{8}$ & $\geq(q-1)\left(q^{2}-q+2\right) / 2$ & $\geq q\left(q^{2}-q+2\right) / 2$ \\
\hline$\chi_{9}$ & $\geq(q-1)\left(q^{2}-q+2\right) / 2$ & $\geq q\left(q^{2}-q+2\right) / 2$ \\
\hline$\chi_{10}$ & $\geq q\left(q^{2}-1\right) / 2$ & $\geq q^{2}(q+1) / 2$ \\
\hline$\chi_{11}$ & $\geq q\left(q^{2}-1\right) / 2$ & $\geq q^{2}(q+1) / 2$ \\
\hline$\chi_{12}$ & $\geq(q-1)^{2}(q+2) / 2$ & $\geq q^{2}(q-1)$ \\
\hline$\chi_{13}$ & $\geq 2 q(q-1)$ & $\geq 2 q^{2}$ \\
\hline \multicolumn{3}{|c|}{} \\
\hline$\theta_{2}(k)$ & $q(q-1)^{2} / 2$ & $q^{2}(q-1) / 2$ \\
\hline$\theta_{3}(k)$ & $q(q-1)^{2} / 2$ & $q^{2}(q-1) / 2$ \\
\hline
\end{tabular}

Note that the characters $\theta_{2}(k)$ and $\theta_{3}(k)$ are rational, now let $\left|\Gamma\left(\chi_{4}(k)\right)\right|=m$ where $\Gamma\left(\chi_{4}(k)\right)=\Gamma\left(Q\left(\chi_{4}(k)\right): Q\right)=\Gamma\left(Q\left(\chi_{5}(k)\right): Q\right)$.

Now by above table and Definition 2.1 and Table $(I)$ of [6], we have $\min \{d(\chi): \operatorname{Ker} \chi=1\}= \begin{cases}2 m q(q-1) & \text { if } q \geq 4 m+1, \\ \frac{q(q-1)^{2}}{2} & \text { otherwise, }\end{cases}$ $\min \{c(\chi)(1): \operatorname{Ker} \chi=1\}= \begin{cases}2 m q^{2} & \text { if } q \geq 4 m+1, \\ \frac{q^{2}(q-1)}{2} & \text { otherwise. }\end{cases}$ Hence $r(B)= \begin{cases}2 m q(q-1) & \text { if } q \geq 4 m+1, \\ \frac{q(q-1)^{2}}{2} & \text { otherwise }\end{cases}$ and $c(B)= \begin{cases}2 m q^{2} & \text { if } q \geq 4 m+1, \\ \frac{q^{2}(q-1)}{2} & \text { otherwise.W }\end{cases}$

In the following theorem, we constructed the $r(G)$ and $c(G)$ of parabolic subgroup $Q$ of $\operatorname{SP}\left(4,2^{n}\right)$.

\section{Theorem 3.2}

Let $G$ be a maximal parabolic subgroup $P$ or $Q$ of $S P\left(4,2^{n}\right)$, then

$$
\begin{aligned}
& \text { 1) } r(G)=\frac{q(q-1)^{2}}{2} \\
& \text { 2) } c(G)=\frac{q^{2}(q-1)}{2} .
\end{aligned}
$$

Proof. Since the groups $P$ and $Q$ have similar proofs, we will prove only $Q$.In 
order to calculate $r(G)$ and $c(G)$, we need to determine $d(\chi)$ and $c(\chi)(1)$ for all characters that are faithful or $\bigcap_{\chi} \operatorname{Ker} \chi=1$.

Then by Corollary 2.2, Lemmas 2.3,2.4 and Table (III) of [6], for the maximal parabolic subgroup $Q$ we have:

$d\left(\chi_{1}\right)=\left|\Gamma\left(\chi_{1}^{\prime}(k)\right)\right| \chi_{1}^{\prime}(k)(1)+\left|\Gamma\left(\chi_{5}^{\prime}(k)\right)\right| \chi_{5}^{\prime}(k)(1) \geq q\left(q^{2}-1\right)+1$ and $c\left(\chi_{1}\right)(1) \geq q^{3}+q^{2}+1$, $d\left(\chi_{2}\right)=\left|\Gamma\left(\chi_{1}^{\prime}(k)\right)\right| \chi_{1}^{\prime}(k)(1)+\left|\Gamma\left(\chi_{6}^{\prime}(k)\right)\right| \chi_{6}^{\prime}(k)(1) \geq q(q-1)^{2}+1$ and $c\left(\chi_{2}\right)(1) \geq q^{3}-q^{2}+1$, $d\left(\chi_{3}\right)=\left|\Gamma\left(\chi_{1}^{\prime}(k)\right)\right| \chi_{1}^{\prime}(k)(1)+\left|\Gamma\left(\theta_{2}^{\prime}(k)\right)\right| \theta_{2}^{\prime}(k)(1) \geq \frac{q\left(q^{2}-1\right)}{2}+1$ and $c\left(\chi_{3}\right)(1) \geq \frac{\left(q^{3}+q^{2}+2\right)}{2}$, $d\left(\chi_{4}\right)=\left|\Gamma\left(\chi_{1}^{\prime}(k)\right)\right| \chi_{1}^{\prime}(k)(1)+\left|\Gamma\left(\theta_{3}^{\prime}(k)\right)\right| \theta_{3}^{\prime}(k)(1) \geq \frac{q(q-1)^{2}}{2}+1$ and $c\left(\chi_{4}\right)(1) \geq \frac{\left(q^{3}-q^{2}+2\right)}{2}$, $d\left(\chi_{5}\right)=\left|\Gamma\left(\chi_{2}^{\prime}(k)\right)\right| \chi_{2}^{\prime}(k)(1)+\left|\Gamma\left(\chi_{5}^{\prime}(k)\right)\right| \chi_{5}^{\prime}(k)(1) \geq q^{3}$ and $c\left(\chi_{5}\right)(1) \geq q^{3}+q^{2}+q+1$, $d\left(\chi_{6}\right)=\left|\Gamma\left(\chi_{2}^{\prime}(k)\right)\right| \chi_{2}^{\prime}(k)(1)+\left|\Gamma\left(\chi_{6}^{\prime}(k)\right)\right| \chi_{6}^{\prime}(k)(1) \geq q\left(q^{2}-2 q+2\right)$ and $c\left(\chi_{6}\right)(1) \geq q^{3}-q^{2}+q+1$, $d\left(\chi_{7}\right)=\left|\Gamma\left(\chi_{2}^{\prime}(k)\right)\right| \chi_{2}^{\prime}(k)(1)+\left|\Gamma\left(\theta_{2}^{\prime}(k)\right)\right| \theta_{2}^{\prime}(k)(1) \geq \frac{q\left(q^{2}+1\right)}{2}$ and $c\left(\chi_{7}\right)(1) \geq \frac{\left(q^{3}+q^{2}+2 q+2\right)}{2}$, $d\left(\chi_{8}\right)=\left|\Gamma\left(\chi_{2}^{\prime}(k)\right)\right| \chi_{2}^{\prime}(k)(1)+\left|\Gamma\left(\theta_{3}^{\prime}(k)\right)\right| \theta_{3}^{\prime}(k)(1) \geq \frac{q\left(q^{2}-2 q+3\right)}{2}$ and $c\left(\chi_{8}\right)(1) \geq \frac{\left(q^{3}-q^{2}+2 q+2\right)}{2}$, $d\left(\chi_{9}\right)=\left|\Gamma\left(\chi_{3}^{\prime}(k, l)\right)\right| \chi_{3}^{\prime}(k, l)(1)+\left|\Gamma\left(\chi_{5}^{\prime}(k)\right)\right| \chi_{5}^{\prime}(k)(1) \geq(q+1)\left(q^{2}-q+1\right)$ and $c\left(\chi_{9}\right)(1) \geq q^{3}+q^{2}+q+2$, $d\left(\chi_{10}\right)=\left|\Gamma\left(\chi_{3}^{\prime}(k, l)\right)\right| \chi_{3}^{\prime}(k, l)(1)+\left|\Gamma\left(\chi_{6}^{\prime}(k)\right)\right| \chi_{6}^{\prime}(k)(1) \geq(q+1)+q(q-1)^{2}$ and $c\left(\chi_{10}\right)(1) \geq q^{3}-q^{2}+q+2$, $d\left(\chi_{11}\right)=\left|\Gamma\left(\chi_{3}^{\prime}(k, l)\right)\right| \chi_{3}^{\prime}(k, l)(1)+\left|\Gamma\left(\theta_{2}^{\prime}(k)\right)\right| \theta_{2}^{\prime}(k)(1) \geq(q+1)\left(q^{2}-q+2\right) / 2$ and $c\left(\chi_{11}\right)(1) \geq \frac{\left(q^{3}+q^{2}+2 q+4\right)}{2}$, $d\left(\chi_{12}\right)=\left|\Gamma\left(\chi_{3}^{\prime}(k, l)\right)\right| \chi_{3}^{\prime}(k, l)(1)+\left|\Gamma\left(\theta_{3}^{\prime}(k)\right)\right| \theta_{3}^{\prime}(k)(1) \geq \frac{(q+1)+q(q-1)^{2}}{2}$ and $c\left(\chi_{12}\right)(1) \geq \frac{\left(q^{3}+q^{2}+2 q+4\right)}{2}$, $d\left(\chi_{13}\right)=\Gamma\left(\chi_{4}^{\prime}(k)\right)\left|\chi_{4}^{\prime}(k)(1)+\right| \Gamma\left(\chi_{5}^{\prime}(k)\right) \mid \chi_{5}^{\prime}(k)(1) \geq\left(q^{2}-1\right)(q+1)$ and $c\left(\chi_{13}\right)(1) \geq q^{2}(q+2)$, $d\left(\chi_{14}\right)=\left|\Gamma\left(\chi_{4}^{\prime}(k)\right)\right| \chi_{4}^{\prime}(k)(1)+\left|\Gamma\left(\chi_{6}^{\prime}(k)\right)\right| \chi_{6}^{\prime}(k)(1) \geq(q-1)\left(q^{2}+1\right)$ and $c\left(\chi_{14}\right)(1) \geq q^{3}$, $d\left(\chi_{15}\right)=\left|\Gamma\left(\chi_{4}^{\prime}(k)\right)\right| \chi_{4}^{\prime}(k)(1)+\left|\Gamma\left(\theta_{2}^{\prime}(k)\right)\right| \theta_{2}^{\prime}(k)(1) \geq(q+2)\left(q^{2}-1\right) / 2 \quad$ and $c\left(\chi_{15}\right)(1) \geq \frac{q^{2}(q+3)}{2}$ $d\left(\chi_{16}\right)=\Gamma\left(\chi_{4}^{\prime}(k)\right)\left|\chi_{4}^{\prime}(k)(1)+\right| \Gamma\left(\theta_{3}^{\prime}(k)\right) \mid \theta_{3}^{\prime}(k)(1) \geq \frac{(q-1)\left(q^{2}+q+2\right)}{2}$ and $c\left(\chi_{16}\right)(1) \geq \frac{q^{2}(q+1)}{2}$ $d\left(\chi_{17}\right)=\left|\Gamma\left(\chi_{7}^{\prime}(k, l)\right)\right| \chi_{7}^{\prime}(k, l)(1)+\left|\Gamma\left(\chi_{5}^{\prime}(k)\right)\right| \chi_{5}^{\prime}(k)(1) \geq(q-1)\left(q^{2}+q+1\right)$ and $c\left(\chi_{17}\right)(1) \geq q\left(q^{2}+q+1\right)$, $d\left(\chi_{18}\right)=\left|\Gamma\left(\chi_{7}^{\prime}(k, l)\right)\right| \chi_{7}^{\prime}(k, l)(1)+\left|\Gamma\left(\chi_{6}^{\prime}(k)\right)\right| \chi_{6}^{\prime}(k)(1) \geq(q-1)\left(q^{2}-q+1\right)$ and $c\left(\chi_{18}\right)(1) \geq q\left(q^{2}-q+1\right)$, $d\left(\chi_{19}\right)=\left|\Gamma\left(\chi_{7}^{\prime}(k, l)\right)\right| \chi_{7}^{\prime}(k, l)(1)+\left|\Gamma\left(\theta_{2}^{\prime}(k)\right)\right| \theta_{2}^{\prime}(k)(1) \geq \frac{(q-1)\left(q^{2}+q+2\right)}{2}$ and $c\left(\chi_{19}\right)(1) \geq \frac{q^{2}(q+3)}{2}$, $d\left(\chi_{20}\right)=\left|\Gamma\left(\chi_{7}^{\prime}(k, l)\right)\right| \chi_{7}^{\prime}(k, l)(1)+\left|\Gamma\left(\theta_{3}^{\prime}(k)\right)\right| \theta_{3}^{\prime}(k)(1) \geq \frac{(q-1)\left(q^{2}-q+2\right)}{2}$ and $c\left(\chi_{20}\right)(1) \geq \frac{q\left(q^{2}-q+2\right)}{2}$, $d\left(\chi_{21}\right)=\left|\Gamma\left(\theta_{1}^{\prime}\right)\right| \theta_{1}^{\prime}(1)+\left|\Gamma\left(\chi_{5}^{\prime}(k)\right)\right| \chi_{5}^{\prime}(k)(1) \geq(2 q-1)\left(q^{2}-1\right)$ and $c\left(\chi_{21}\right)(1) \geq 2\left(q^{3}+q^{2}-1\right)$, $d\left(\chi_{22}\right)=\left|\Gamma\left(\theta_{1}^{\prime}\right)\right| \theta_{1}^{\prime}(1)+\left|\Gamma\left(\chi_{6}^{\prime}(k)\right)\right| \chi_{6}^{\prime}(k)(1) \geq(2 q+1)(q-1)^{2}$ and $c\left(\chi_{22}\right)(1) \geq 2 q^{2}(q-1)$, 


$$
\begin{aligned}
& d\left(\chi_{23}\right)=\left|\Gamma\left(\theta_{1}^{\prime}\right)\right| \theta_{1}^{\prime}(1)+\left|\Gamma\left(\theta_{2}^{\prime}(k)\right)\right| \theta_{2}^{\prime}(k)(1) \geq \frac{(3 q-2)\left(q^{2}-1\right)}{2} \quad \text { and } c\left(\chi_{23}\right)(1) \geq \frac{q^{2}(3 q-1)}{2}, \\
& d\left(\chi_{24}\right)=\mid \Gamma\left(\left(\theta_{1}^{\prime}\right)\left|\theta_{1}^{\prime}(1)+\right| \Gamma\left(\theta_{3}^{\prime}(k)\right) \mid \theta_{3}^{\prime}(k)(1) \geq \frac{(3 q+2)(q-1)^{2}}{2} \text { and } c\left(\chi_{24}\right)(1) \geq \frac{3 q^{2}(q-1)}{2},\right. \\
& d\left(\chi_{5}^{\prime}(k)\right)=\left|\Gamma\left(\chi_{5}^{\prime}(k)\right)\right| \chi_{5}^{\prime}(k)(1) \geq q\left(q^{2}-1\right) \text { and } c\left(\chi_{5}^{\prime}(k)\right)(1) \geq q^{2}(q+1), \\
& d\left(\chi_{6}^{\prime}(k)\right)=\left|\Gamma\left(\chi_{6}^{\prime}(k)\right)\right| \chi_{6}^{\prime}(k)(1) \geq q(q-1)^{2} \quad \text { and } c\left(\chi_{6}^{\prime}(k)\right)(1) \geq q^{2}(q-1), \\
& d\left(\theta_{2}^{\prime}(k)\right)=\left|\Gamma\left(\theta_{2}^{\prime}(k)\right)\right| \theta_{2}^{\prime}(k)(1)=\frac{q\left(q^{2}-1\right)}{2} \text { and } c\left(\theta_{2}^{\prime}(k)\right)(1)=\frac{q^{2}(q+1)}{2}, \\
& d\left(\theta_{3}^{\prime}(k)\right)=\left|\Gamma\left(\theta_{3}^{\prime}(k)\right)\right| \theta_{3}^{\prime}(k)(1)=\frac{q(q-1)^{2}}{2} \text { and } c\left(\theta_{3}^{\prime}(k)\right)(1)=\frac{q^{2}(q-1)}{2} .
\end{aligned}
$$

\begin{tabular}{|c|c|c|}
\hline$\chi$ & $d(\chi)$ & $c(\chi)(1)$ \\
\hline$\chi_{1}$ & $\geq q\left(q^{2}-1\right)+1$ & $\geq q^{3}+q^{2}+1$ \\
\hline$\chi_{2}$ & $\geq q(q-1)^{2}+1$ & $\geq q^{3}-q^{2}+1$ \\
\hline$\chi_{3}$ & $\geq q\left(q^{2}-1\right) / 2+1$ & $\geq\left(q^{3}+q^{2}+2\right) / 2$ \\
\hline$\chi_{4}$ & $\geq q(q-1)^{2} / 2+1$ & $\geq\left(q^{3}-q^{2}+2\right) / 2$ \\
\hline$\chi_{5}$ & $\geq q^{3}$ & $\geq q^{3}+q^{2}+q+1$ \\
\hline$\chi_{6}$ & $\geq q\left(q^{2}-2 q+2\right)$ & $\geq q^{3}-q^{2}+q+1$ \\
\hline$\chi_{7}$ & $\geq q\left(q^{2}+1\right) / 2$ & $\geq\left(q^{3}+q^{2}+2 q+2\right) / 2$ \\
\hline$\chi_{8}$ & $\geq q\left(q^{2}-2 q+3\right) / 2$ & $\geq\left(q^{3}-q^{2}+2 q+2\right) / 2$ \\
\hline$\chi_{9}$ & $\geq(q+1)\left(q^{2}-q+1\right)$ & $\geq q^{3}+q^{2}+q+2$ \\
\hline$\chi_{10}$ & $\geq(q+1)+q(q-1)^{2}$ & $\geq q^{3}-q^{2}+q+2$ \\
\hline$\chi_{11}$ & $\geq(q+1)\left(q^{2}-q+2\right) / 2$ & $\geq\left(q^{3}+q^{2}+2 q+4\right) / 2$ \\
\hline$\chi_{12}$ & $\geq(q+1)+q(q-1)^{2} / 2$ & $\geq\left(q^{3}-q^{2}+2 q+4\right) / 2$ \\
\hline$\chi_{13}$ & $\geq(q+1)\left(q^{2}-1\right)$ & $\geq q^{2}(q+2)$ \\
\hline$\chi_{14}$ & $\geq(q-1)\left(q^{2}+1\right)$ & $\geq q^{3}$ \\
\hline$\chi_{15}$ & $\geq(q+2)\left(q^{2}-1\right) / 2$ & $\geq q^{2}(q+3) / 2$ \\
\hline$\chi_{16}$ & $\geq(q-1)\left(q^{2}+q+2\right) / 2$ & $\geq q^{2}(q+1) / 2$ \\
\hline$\chi_{17}$ & $\geq(q-1)\left(q^{2}+q+1\right)$ & $\geq q\left(q^{2}+q+1\right)$ \\
\hline$\chi_{18}$ & $\geq(q-1)\left(q^{2}-q+1\right)$ & $\geq q\left(q^{2}-q+1\right)$ \\
\hline$\chi_{19}$ & $\geq(q-1)\left(q^{2}+q+2\right) / 2$ & $\geq q^{2}(q+3) / 2$ \\
\hline$\chi_{20}$ & $\geq(q-1)\left(q^{2}-q+2\right) / 2$ & $\geq q\left(q^{2}-q+2\right) / 2$ \\
\hline$\chi_{21}$ & $\geq(2 q-1)\left(q^{2}-1\right)$ & $\geq 2\left(q^{3}+q^{2}-1\right)$ \\
\hline
\end{tabular}

The values are set out in the following table:

Table (II) 


\begin{tabular}{|c|c|c|}
\hline$\chi_{22}$ & $\geq(2 q+1)(q-1)^{2}$ & $\geq 2 q^{2}(q-1)$ \\
\hline$\chi_{23}$ & $\geq(3 q-2)\left(q^{2}-1\right) / 2$ & $\geq q^{2}(3 q-1) / 2$ \\
\hline$\chi_{24}$ & $\geq(3 q+2)(q-1)^{2} / 2$ & $\geq 3 q^{2}(q-1) / 2$ \\
\hline$\chi_{5}^{\prime}(k)$ & $\geq q\left(q^{2}-1\right)$ & $\geq q^{2}(q+1)$ \\
\hline$\chi_{6}^{\prime}(k)$ & $\geq q(q-1)^{2}$ & $\geq q^{2}(q-1)$ \\
\hline$\theta_{2}^{\prime}(k)$ & $q\left(q^{2}-1\right) / 2$ & $q^{2}(q+1) / 2$ \\
\hline$\theta_{3}^{\prime}(k)$ & $q(q-1)^{2} / 2$ & $q^{2}(q-1) / 2$ \\
\hline
\end{tabular}

Now by Table (II) and Definition 2.1 ,we have $\min \{d(\chi): \operatorname{Ker} \chi=1\}=\frac{q(q-1)^{2}}{2}$ and $\min \{c(\chi)(1): \operatorname{Ker} \chi=1\}=\frac{q^{2}(q-1)}{2}$.

Hence $r(G)=\frac{q(q-1)^{2}}{2}, c(G)=\frac{q^{2}(q-1)}{2}, \quad$ and the result follows.

\section{References}

[1] H. Behravesh, Quasi-permutation representations of p-groups of class 2 , J.London Math. Soc. (2)55, 251-26 (1997).

[2] J.M. Burns , B. Goldsmith , B. Hartley and R. Sandling, on quasi-permutation representations of finite groups, Glasgow Math, J. 36 , 301-308 (1994).

[3] M.R. Darafsheh, M. Ghorbany , A. Daneshkhah and H. Behravesh, Quasi-permutation representation of the group $G L(2, q)$, Journal of Algebra 243,142-167(2001).

[4] M.R. Darafsheh and M.Ghorbany, Quasi-permutation representations of the groups $S U\left(3, q^{2}\right)$ and $\operatorname{PSU}\left(3, q^{2}\right)$, Southest Asian Bulletin of Mathemetics (2002)26 ,395-406.

[5] M.R. Darafsheh and M.Ghorbany, Quasi-permutation representations of the groups $S L(3, q)$ and $P S L(3, q)$, Iranian Journal of Science and Technology, Tra.A, Vol. 26, No. A1 (2002)145-154.

[6] H. Enomoto, The characters of the finite symplectic group $S P(4, q), q=2^{f}$, Osaka J. Math. 9(1972), 75-94. 
[7] M.R. Darafsheh and M.Ghorbany, Special representations of the group $G_{2}\left(2^{n}\right)$ with minimal degrees, Southest Asian Bulletin of Mathemetics (2006)30: 663-670.

[8] W.J. Wong, Linear groups analogous to permutation groups, J. Austral. Math. Soc (Sec. A) $3,180-184$, (1963). 